\title{
Utilisation des isotopes de l'uranium et du rapport U/Th pour évaluer le marquage des végétaux consécutif aux rejets d'uranium des installations du cycle du combustible
}

\author{
L. Pourcelot ${ }^{1 \star}$, B. Boulet ${ }^{2}$ et N. Cariou ${ }^{2}$ \\ 1 IRSN/PRP-ENV/SESURE/LERCM, Bât. 153, BP 3, 13115 Saint-Paul-lez-Durance Cedex, France. \\ 2 IRSN/PRP-ENV/STEME/LMRE, Bât. 501, Bois des Rames, 91400 Orsay Cedex, France.
}

Reçu le 10 mars 2015 - Accepté le 20 mai 2015

\begin{abstract}
Résumé - Cette note s'appuie sur des données de la surveillance de l'environnement des installations du cycle du combustible. Il s'agit d'évaluer l'uranium ajouté dans l'environnement terrestre par les rejets. Ainsi, nous illustrons par la mesure des isotopes de l'uranium dans les plantes terrestres les conséquences d'un rejet chronique et d'un rejet incidentel d'uranium appauvri dans l'atmosphère. Cependant, cette méthode analytique atteint ses limites quand les rejets des installations concernent l'uranium naturel. Distinguer l'uranium naturel provenant des rejets et l'uranium qui dérive du fond radiologique devient alors délicat. C'est pourquoi, nous proposons de normaliser l'activité en uranium des plantes prélevées autour des sites nucléaires par rapport à l'activité en ${ }^{232} \mathrm{Th}$, en considérant que la source de ce dernier est le fond radiologique.
\end{abstract}

\begin{abstract}
The use of uranium isotopes and the U/Th ratio to evaluate the fingerprint of plants following uranium releases from fuel cycle settlements. This paper uses data from the environmental monitoring of fuel cycle settlements. It aims to evaluate uranium released into the terrestrial environment. Measurement of uranium isotopes in terrestrial plants allows illustrating the consequences of chronic and incidental releases of depleted uranium into the atmosphere. However, such an analytical approach reaches its limits when natural uranium is released. Indeed, distinguishing natural uranium from releases and uranium from the radiological background is difficult. For this reason, we propose normalizing uranium activity measured in plants taken in the surroundings of nuclear sites with respect to ${ }^{232} \mathrm{Th}$, considering that the source of this latter is the background.
\end{abstract}

Keywords: uranium / depleted uranium / plants / discharges / thorium

\section{Introduction}

Les conséquences des rejets en uranium provenant des installations du cycle du combustible dans l'environnement peuvent être évaluées par les rapports des isotopes de l'uranium. Ainsi, l'activité des isotopes majeurs $\left({ }^{238} \mathrm{U},{ }^{234} \mathrm{U}\right)$ et mineurs de l'uranium $\left({ }^{235} \mathrm{U},{ }^{236} \mathrm{U}\right)$ est mesurée dans différentes matrices prélevées dans l'environnement, principalement des sols, des sédiments, des végétaux terrestres et aquatiques. Les rapports de ces isotopes, véritable «empreinte génétique » de l'uranium, permettent d'évaluer l'uranium ajouté dans l'environnement par les rejets provenant des installations (Pourcelot et al., 2011a). En effet, les variations des rapports ${ }^{235} \mathrm{U} /{ }^{238} \mathrm{U}$ et ${ }^{236} \mathrm{U} /{ }^{238} \mathrm{U}$ en fonction de l'activité en uranium dans l'environnement peuvent permettre de distinguer les deux

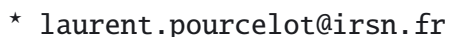

sources principales de l'uranium : l'uranium provenant des rejets des installations, lorsque les process industriels modifient les rapports isotopiques de l'uranium et l'uranium provenant du fond radiologique. Ainsi, l'augmentation de l'activité en uranium est proportionnelle à l'anomalie du rapport massique ${ }^{235} \mathrm{U} /{ }^{238} \mathrm{U}$ (la valeur du fond radiologique est de $0,72 \%$ ), attestant, selon le cas, de la présence d'uranium appauvri ou d'uranium enrichi (Pettersson et Holm, 1992; Bellis et al., 2001; Howe et al., 2002; Pourcelot et al., 2011b). De la même façon, une matrice de l'environnement influencée par les rejets de l'uranium de retraitement (URT) est marquée par la présence de $1{ }^{236} \mathrm{U}$ (isotope artificiel) (Ketterer et al., 2003 ; Lloyd et al., 2009; Pourcelot et al., 2011c; Tortorello et al., 2014).

Cette note s'appuie sur des données de la surveillance de l'environnement des installations du cycle du combustible. Il s'agit d'évaluer l'uranium ajouté dans l'environnement terrestre par les rejets. Ainsi, dans la première partie de cette 
note, nous illustrons par la mesure des isotopes de l'uranium dans les plantes terrestres les conséquences d'un rejet chronique et d'un rejet incidentel d'uranium appauvri dans l'atmosphère. Cependant, cette méthode analytique atteint ses limites quand les rejets des installations concernent l'uranium naturel. Distinguer l'uranium naturel provenant des rejets et l'uranium qui dérive du fond radiologique devient alors délicat. C'est pourquoi, dans la seconde partie de cette note, nous proposons de normaliser l'activité en uranium des plantes prélevées autour des sites nucléaires par rapport à l'activité en ${ }^{232} \mathrm{Th}$, en considérant que la source de ce dernier est le fond radiologique.

\section{Origine des données}

\section{1 Échantillonnage dans l'environnement des installations}

Les données proviennent des études menées par l'IRSN dans l'environnement de deux sites AREVA du cycle du combustible. Le site de Malvési, localisé au nord de Narbonne (11), produit de l' $\mathrm{UF}_{4}$ (environ $12000 \mathrm{t}^{\mathrm{a}} \mathrm{an}^{-1}$ ) après purification et fluoration de l'uranium naturel, initialement sous la forme de «yellow cake ». Cette activité génère des rejets atmosphériques en uranium évalués entre 102 et $18 \mathrm{~kg} \cdot \mathrm{an}^{-1}$, entre 2007 et 2013 (AREVA, 2010, 2013a). À titre exceptionnel, 7200 tonnes d'uranium appauvri ont été converties en $\mathrm{UF}_{4}$ sur le site de Malvési en 2008. L'IRSN dispose de prélèvements et de mesures de plantes (10 échantillons de grains de blé, 4 échantillons de légumes feuilles et 6 échantillons de feuilles de cyprès) réalisés entre 2007 et 2013 dans la zone influencée par les rejets atmosphériques (est du site), à des distances variant entre 50 et 1500 mètres du site (Pourcelot et al., 2015).

Le site de Tricastin, localisé dans la vallée du Rhône à proximité de Pierrelatte (26), comporte plusieurs installations de traitement de l'uranium (conversion, enrichissement et défluoration, principalement). Ces activités génèrent des rejets chroniques dans l'atmosphère plus faibles que ceux du site de Malvési, de l'ordre de 2 kg.an ${ }^{-1}$ entre 2011 et 2013 (AREVA, 2013b). Autour du site, des végétaux (5 échantillons de grains de blé et 8 échantillons d'herbe) ont été prélevés en 2012 par l'IRSN, à des distances comprises entre 1000 et 1500 mètres environ de l'enceinte du site. De nouveaux prélèvements ont été effectués par l'IRSN en janvier 2014 (4 échantillons d'herbe, 1 échantillon de feuilles de cyprès et 1 échantillon de feuilles de chêne), suite à un rejet incidentel d'uranium appauvri survenu sur l'installation W (défluoration de l' $\mathrm{UF}_{6}$ en $\mathrm{U}_{3} \mathrm{O}_{8}$ ), dans le but d'évaluer les conséquences de ce rejet dans l'environnement terrestre proche du site. Ainsi, deux prélèvements d'herbe proviennent du nord et du sud du site (environ 3000 mètres du point de rejet). Les autres végétaux (2 échantillons d'herbe et 2 de feuilles d'arbres) ont été échantillonnés à l'est du site, à 600 et 800 mètres environ du point de rejet.

Les échantillons de végétaux prélevés (1-3 kg de matière fraîche) sont séchés à $80^{\circ} \mathrm{C}$ avant incinération. Seuls les légumes feuilles ont été lavés au préalable.

\subsection{Métrologie des isotopes de l'uranium et du thorium}

Les échantillons ont été mis en solution par digestion acide en système ouvert avec le DigiPREP Jr (SCP Science $\left.{ }^{\circledR}\right)$. Les prises d'essai étaient comprises entre 0,2 et $0,8 \mathrm{~g}$ (cendre) et les réactifs, de qualité Suprapur, étaient $\mathrm{HNO}_{3}, \mathrm{H}_{2} \mathrm{O}_{2}, \mathrm{HF}$ et $\mathrm{HCl}$. U et Th ont ensuite été séparés sur résine UTEVA (Triskem ${ }^{\circledR}$ ) puis les isotopes de chaque élément ont été mesurés par ICP-QMS (XSeries2, ThermoFisher Scientific). Le système d'introduction de l'ICP-MS se composait d'un nébuliseur concentrique (Meinhard ${ }^{\mathbb{Q}}$ ou Arimist ${ }^{\mathbb{R}}, 400 \mu$ L.min ${ }^{-1}$ ) et d'une chambre cyclonique refroidie par effet Peltier. Les activités des isotopes de $\mathrm{U}$ et de $\mathrm{Th}$, ainsi que leurs ratios, ont été déterminés avec la méthode de la dilution isotopique en ajoutant les traceurs ${ }^{233} \mathrm{U}$ et ${ }^{229} \mathrm{Th}$ dès le début de la minéralisation des échantillons. La correction du biais en masse a également été effectuée selon le modèle linéaire.

Enfin, un blanc a été réalisé dans chaque série d'échantillons et des échantillons de référence certifiés ont été régulièrement analysés.

Dans la suite de cette note, l'activité massique des radionucléides dans les végétaux est exprimée en $\mathrm{Bq} \cdot \mathrm{kg}^{-1}$ frais, à l'exception des grains de blé échantillonnés à maturité (c'est-à-dire quasiment secs), dont l'activité est exprimée en Bq. $\mathrm{kg}^{-1} \mathrm{sec}$. Les incertitudes des activités représentées sur les figures sont les incertitudes analytiques.

\section{Résultats et discussion}

\section{1 Évaluation du marquage des végétaux consécutif aux rejets d'uranium appauvri dans l'environnement terrestre}

En ce qui concerne les données provenant de l'environnement de Tricastin, d'importantes variations de l'activité en ${ }^{238} \mathrm{U}$ et du rapport de masse ${ }^{235} \mathrm{U} /{ }^{238} \mathrm{U}$ sont observées dans les végétaux lorsque les échantillons prélevés en 2014 (après le rejet incidentel d'uranium appauvri) sont comparés à ceux de 2012 (avant le rejet) (Figure 1). Ainsi, les végétaux prélevés en 2014 témoignent de l'activité en ${ }^{238} \mathrm{U}$ la plus élevée $(0,1 \pm 0,01$ à $\left.4 \pm 0,4 \mathrm{~Bq} \cdot \mathrm{kg}^{-1}\right)$ et du rapport ${ }^{235} \mathrm{U} /{ }^{238} \mathrm{U}$ le plus faible $(0,32 \pm$ $0,01$ à $0,43 \pm 0,02 \%)$, en comparaison de l'activité et du rapport observés en $2012\left(0,01 \pm 0,002\right.$ à $0,02 \pm 0,002 \mathrm{~Bq} \cdot \mathrm{kg}^{-1}$ et $0,72 \pm 0,02 \%$, respectivement). Ces dernières données sont proches de l'activité en uranium mesurée dans ces matrices prélevées en dehors de l'influence des rejets de l'industrie nucléaire et du rapport ${ }^{235} \mathrm{U} /{ }^{238} \mathrm{U}$ observé dans la nature $(0,72 \%)$ (Jeambrun et al., 2012).

Concernant les données acquises dans l'environnement de Malvési, une augmentation de l'activité en ${ }^{238} \mathrm{U}(0,3 \pm 0,03$ à $7 \pm 1 \mathrm{~Bq} \cdot \mathrm{kg}^{-1}$ ) et un rapport ${ }^{235} \mathrm{U} /{ }^{238} \mathrm{U}$ inférieur à $0,72 \%$ $(0,39 \pm 0,02$ à $0,48 \pm 0,02 \%)$ sont observés dans les grains de blé prélevés en 2008, lorsque l'installation de Malvési convertissait de l'uranium appauvri (Pourcelot et al., 2011b). La comparaison avec le rapport isotopique du sol de culture $(0,72 \%)$ a montré que l'uranium appauvri rejeté de manière chronique par voie atmosphérique contribue à la contamination des plantes dans une proportion variable selon la distance qui sépare l'échantillon du point de rejet, alors que la contribution du sol est négligeable (Pourcelot et al., 2011b). 


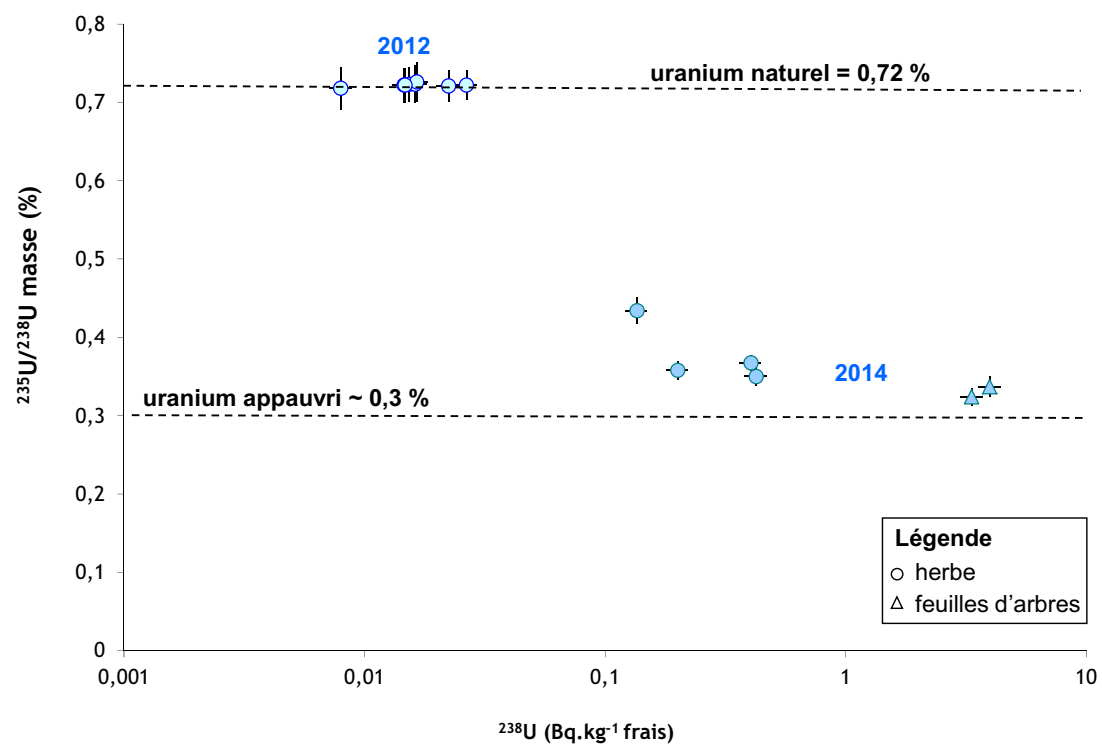

Figure 1. Rapport de masse ${ }^{235} \mathrm{U} /{ }^{238} \mathrm{U}$ (en \%) en fonction de l'activité en ${ }^{238} \mathrm{U}$ (en Bq.kg-1 frais) dans les végétaux terrestres prélevés dans l'environnement des installations AREVA de Tricastin en 2012 (avant le rejet incidentel) et en 2014 (après le rejet incidentel).

${ }^{235} \mathrm{U} /{ }^{238} \mathrm{U}$ mass ratio (\%) with respect to ${ }^{238} \mathrm{U}$ activity (Bq.kg ${ }^{-1}$ fresh weight) in terrestrial plants taken in the environment of the AREVA nuclear settlement of Tricastin in 2012 (before the accidental release) and in 2014 (after the nuclear release).

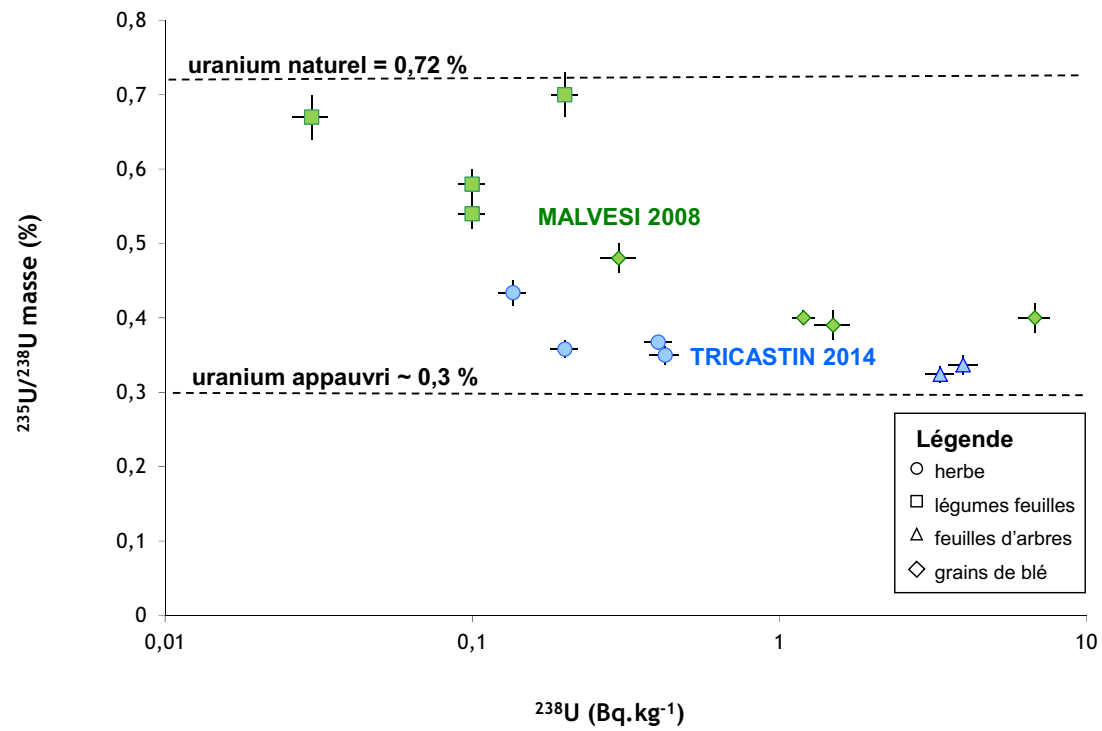

Figure 2. Rapport de masse ${ }^{235} \mathrm{U} /{ }^{238} \mathrm{U}$ (en \%) en fonction de l'activité en ${ }^{238} \mathrm{U}$ (en Bq. $\mathrm{kg}^{-1}$ frais, à l'exception des grains de blé, dont l'activité est exprimée en $\mathrm{Bq} \cdot \mathrm{kg}^{-1} \mathrm{sec}$ ) dans les végétaux terrestres prélevés dans l'environnement des installations AREVA de Malvési (rejet chronique d'uranium appauvri en 2008) et de Tricastin (rejet incidentel d'uranium appauvri fin 2013).

${ }^{235} \mathrm{U} /{ }^{238} \mathrm{U}$ mass ratio (in \%) with respect to ${ }^{238} \mathrm{U}$ activity in terrestrial plants (in $\mathrm{Bq} \cdot \mathrm{kg}^{-1}$ fresh weight, with the exception of wheat grains, whose activity is expressed in Bq. $\mathrm{kg}^{-1}$ dry matter) taken in the environment of the AREVA nuclear settlements of Malvési (chronic release in 2008) and Tricastin (accidental release at the end of 2013).

Sur la Figure 2 est illustré le marquage des végétaux par l'uranium, conséquence du rejet chronique d'uranium appauvri (site de Malvési, 2008) et du rejet incidentel d'uranium appauvri (site de Tricastin, 2013). L'activité en uranium est globalement comparable dans les deux cas présentés. Cependant, les végétaux prélevés à Tricastin témoignent d'un rapport massique ${ }^{235} \mathrm{U}^{238} \mathrm{U}$ plus faible que les échantillons issus de Malvési, probablement parce que la signature de l'uranium appauvri rejeté n'est pas la même dans les deux cas.

\section{2 Évaluation du marquage des végétaux consécutif aux rejets d'uranium naturel en milieu terrestre, par l'utilisation du rapport d'activité ${ }^{238} \mathrm{U} /{ }^{232} \mathrm{Th}$}

Pour évaluer le marquage des végétaux consécutifs aux rejets d'uranium naturel dans l'environnement des sites du cycle du combustible, l'activité en uranium des végétaux prélevés dans l'environnement des deux sites du cycle du combustible a été normalisée par rapport à l'activité en ${ }^{232} \mathrm{Th}$. 


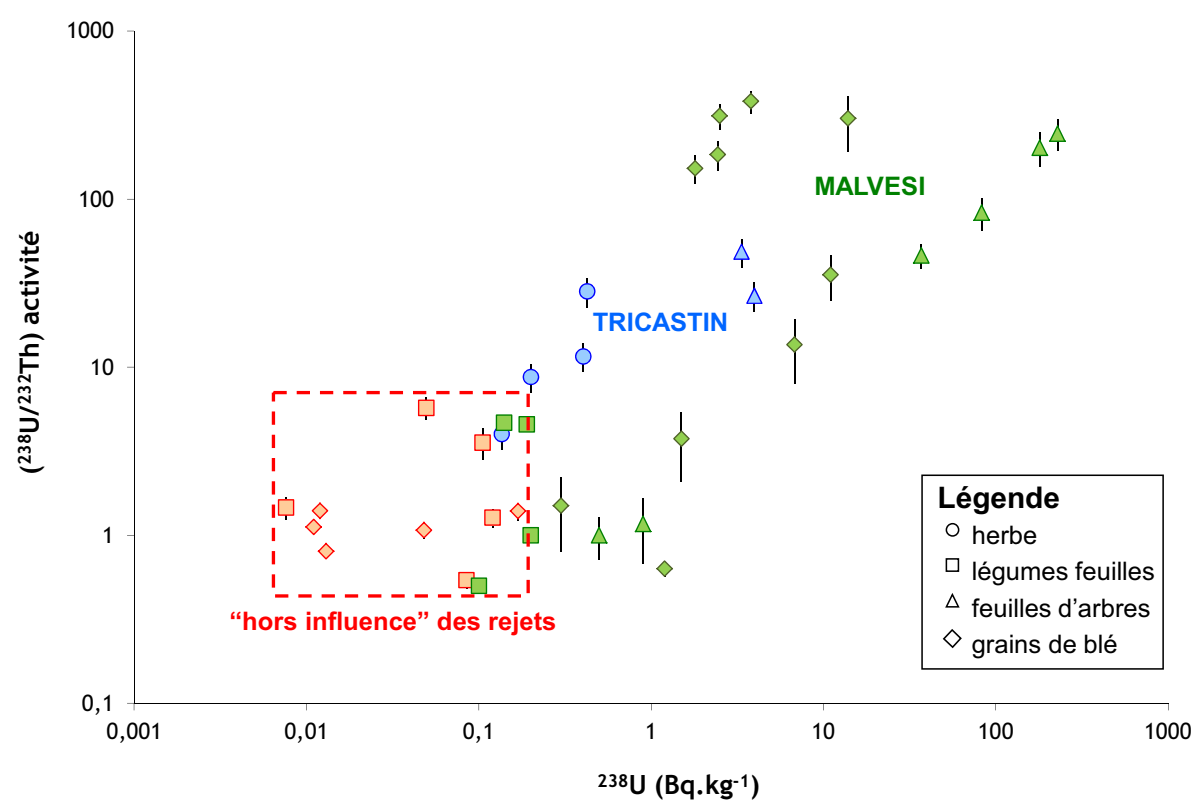

Figure 3. Rapport d'activité ${ }^{238} \mathrm{U} /{ }^{232} \mathrm{Th}$ en fonction de l'activité en ${ }^{238} \mathrm{U}$ (en Bq.kg ${ }^{-1}$ frais, à l'exception des grains de blé, dont l'activité est exprimée en Bq. $\mathrm{kg}^{-1} \mathrm{sec}$ ) dans les végétaux terrestres prélevés dans l'environnement des installations AREVA de Malvési et de Tricastin. Ces données sont comparées à celles d'échantillons prélevés en dehors de l'influence des rejets des installations nucléaires (Jeambrun et al., 2012). ${ }^{238} \mathrm{U} /{ }^{332} \mathrm{Th}$ activity ratio with respect to ${ }^{238} \mathrm{U}$ activity (in Bq. $\mathrm{kg}^{-1}$ fresh material, with the exception of wheat grains, whose activity is expressed in Bq. $\mathrm{kg}^{-1}$ dry matter) in terrestrial plants taken in the environment of the AREVA nuclear settlements of Malvési and Tricastin. These data are compared with those of samples taken outside the influence of the releases from nuclear settlements (after Jeambrun et al., 2012).

La normalisation de l'activité en ${ }^{238} \mathrm{U}$ par un élément qui dérive uniquement des sols $\left({ }^{232} \mathrm{Th}\right)$ doit permettre de distinguer les végétaux qui possèdent une signature comparable à cette source (rapport d'activité ${ }^{238} \mathrm{U} /{ }^{232} \mathrm{Th}$ des sols proche de la valeur unitaire, d'après Le Roux, 2007) et, par ailleurs, les végétaux qui présentent un excès d'uranium provenant des rejets. À cette fin, les rapports d'activités ${ }^{238} \mathrm{U} /{ }^{232} \mathrm{Th}$ des végétaux issus de l'environnement des sites nucléaires sont comparés aux rapports des végétaux échantillonnés dans des zones situées en dehors de l'influence des rejets de l'industrie nucléaire (Jeambrun et al., 2012) (Figure 3). Ainsi, le lot d'échantillons de référence possède l'activité en ${ }^{238} \mathrm{U}$ et le rapport d'activité les plus faibles (compris entre $0,008 \pm 0,001$ et $0,17 \pm$ $0,02 \mathrm{~Bq} \cdot \mathrm{kg}^{-1}$ et entre $0,5 \pm 0,06$ et $5,7 \pm 0,9$, respectivement). En comparaison de ce lot de données représentant le fond radiologique, certains des échantillons issus de l'environnement des deux sites nucléaires témoignent de l'activité en ${ }^{238}$ U la plus élevée. De plus, l'augmentation de l'activité en uranium s'accompagne d'une augmentation de la proportion de l'uranium par rapport au thorium $\left({ }^{238} \mathrm{U} /{ }^{232} \mathrm{Th}\right)$, marquant ainsi l'excès de l'uranium provenant des rejets par rapport au thorium qui représente le fond radiologique. Notons cependant que quelques échantillons de l'environnement des deux sites témoignent d'une activité en uranium et d'un rapport d'activité comparables à celui du fond radiologique.

\section{Discussion}

Le rapport de masse ${ }^{235} \mathrm{U} /{ }^{238} \mathrm{U}$ atteste sans ambigüité du marquage des végétaux par l'uranium appauvri, qu'il s'agisse d'un rejet de routine ou d'un rejet incidentel. Au-delà de cette observation, ces données permettent de calculer la quantité d'uranium provenant du rejet, en utilisant une équation de mélange (Yoshida et al., 2001; Tortorello et al., 2014). Ainsi, en supposant que le rapport ${ }^{235} \mathrm{U} /{ }^{238} \mathrm{U}$ massique de l'uranium rejeté est de $0,3 \%$, l'activité en ${ }^{238} \mathrm{U}$ ajoutée dans les végétaux par le rejet incidentel de Tricastin varie entre 0,1 et 3,2 Bq. $\mathrm{kg}^{-1}$ selon l'échantillon considéré.

Les données acquises dans des conditions expérimentales proches de la présente étude et provenant de l'environnement d'autres sites du cycle du combustible et de mines d'uranium à travers le monde sont synthétisées dans le Tableau 1. Ces études intègrent également des données représentatives du fond radiologique, provenant de zones situées en dehors de l'influence des rejets de l'industrie nucléaire.

Comme observé pour le lot d'échantillons de référence utilisé dans notre étude (Jeambrun et al., 2012), les plantes provenant de zones non-influencées par les rejets sont caractérisées, quelle que soit leur origine, par l'activité en uranium et le rapport d'activité ${ }^{238} \mathrm{U} /{ }^{232} \mathrm{Th}$ parmi les plus faibles. Le rapport d'activité des végétaux, proche de la valeur unitaire dans la plupart des cas, est compatible avec celui du sol et de la croûte terrestre, principales sources de l'uranium pour les plantes terrestre. En effet, dans ces deux compartiments, l'activité en uranium et en thorium est très proche, de l'ordre de 30 à 40 Bq. kg ${ }^{-1}$ (Taylor, 1964; Wedepohl, 1995; Yoshida et al., 1998; UNSCEAR, 2000 ; Le Roux, 2007). Les activités en uranium et en thorium dans les sols des deux sites nucléaires sont de cet ordre de grandeur, relativement faible et homogène. De plus, une partie des variations de l'activité et du rapport d'activité observées dans le fond radiologique (Tableau 1) provient probablement des différentes espèces végétales étudiées, 
Tableau 1. Activité en ${ }^{238} \mathrm{U}$ et rapport d'activité ${ }^{238} \mathrm{U} /{ }^{232} \mathrm{Th}$ dans les plantes prélevées dans l'environnement de sites du cycle du combustible, de mines d'uranium et dans des sites représentant le fond radiologique (en grisé). Les valeurs moyennes sont données en italique.

Activity of ${ }^{238} \mathrm{U}$ and the ${ }^{238} \mathrm{U} /{ }^{232} \mathrm{Th}$ activity ratio in plants taken in the environment of nuclear fuel sites, uranium mines and sites from the radiological background (in gray). Mean values are given in italics.

\begin{tabular}{|c|c|c|c|c|}
\hline Origine des échantillons & Matrice & ${ }^{238} \mathrm{U}\left(\mathrm{Bq} \cdot \mathrm{kg}^{-1}\right)$ & ${ }^{238} \mathrm{U} /{ }^{232} \mathrm{Th}$ & Référence \\
\hline France & légumes feuilles & 0,05 à 0,12 & 0,5 à 5,7 & Jeambrun et al., 2012 \\
\hline France & grains de blé & 0,01 à 0,17 & 0,8 à 1,4 & Jeambrun et al., 2012 \\
\hline $\begin{array}{l}\text { Usine de conversion de l'uranium, } \\
\text { Malvési (F) }\end{array}$ & $\begin{array}{l}\text { grains de blé, } \\
\text { légumes feuilles } \\
\text { et feuilles d'arbres }\end{array}$ & 0,1 à 228 & 0,5 à 383 & présente étude \\
\hline $\begin{array}{l}\text { Usine de traitement de l'uranium, } \\
\text { Tricastin (F) }\end{array}$ & $\begin{array}{l}\text { herbe, feuilles } \\
\text { d'arbres }\end{array}$ & 0,1 à 3,3 & 4 à 49 & présente étude \\
\hline Forêt des Vosges $(\mathrm{F})$ & feuilles d'arbres & 0,04 & 1,6 & Rihs et al., 2011 \\
\hline Rivelin (GB) & écorce d'arbres & 0,8 & 3,2 & Bellis et al., 2001 \\
\hline $\begin{array}{l}\text { Usine d'enrichissement, } \\
\text { Capenhurst (GB) }\end{array}$ & écorce d'arbres & 4,0 & 18 & Bellis et al., 2001 \\
\hline $\begin{array}{l}\text { Usine de production du combustible, } \\
\text { Springfield (GB) }\end{array}$ & écorce d'arbres & $\begin{array}{l}62 \\
15 \\
4,7\end{array}$ & $\begin{array}{c}620 \\
150 \\
94\end{array}$ & $\begin{array}{l}\text { Bellis et al., } \\
\quad 2001\end{array}$ \\
\hline San Paulo (Brésil) & feuilles d'arbres & 0,15 & 1,8 & Buchmann et al., 2000 \\
\hline $\begin{array}{l}\text { Institut de recherche nucléaire, } \\
\text { San Paulo (Brésil) }\end{array}$ & feuilles d'arbres & $\begin{array}{l}0,7 \\
0,9\end{array}$ & $\begin{array}{c}11 \\
5,3\end{array}$ & $\begin{array}{l}\text { Buchmann et al. } \\
2000\end{array}$ \\
\hline Kurchatov (Kazakhstan) & herbe & 0,18 à 0,77 & 0,6 à 0,84 & Zoriy et al., 2010 \\
\hline $\begin{array}{l}\text { Aktau (Kazaknstan) } \\
\text { Usine de traitement de l'uranium, } \\
\text { Aktau (Kazakhstan) }\end{array}$ & $\begin{array}{l}\text { herbe } \\
\text { herbe }\end{array}$ & 1,4 à 16 & 1,0 à 3,2 & Zoriy et al., 2010 \\
\hline $\begin{array}{l}\text { Mines d'uranium, } \\
\text { Aktau (Kazakhstan) }\end{array}$ & herbe & 1,7 à 16 & 0,8 à 2,3 & Zoriy et al., 2010 \\
\hline
\end{tabular}

qui prélèvent les deux radionucléides des sols dans des proportions différentes. Rappelons que les variations de l'uranium et du thorium dans les plantes prélevées hors influence des rejets sous l'effet de paramètres de l'environnement (espèce végétale étudiée, type de sol, variations saisonnières, etc.) ne sont pas l'objet de cette note. Enfin, en comparaison des échantillons issus des sites représentant le fond radiologique, l'activité en ${ }^{238} \mathrm{U}$ et le rapport d'activité ${ }^{238} \mathrm{U} /{ }^{232} \mathrm{Th}$ sont plus élevés à proximité des sites du cycle du combustible et des mines d'uranium (Tableau 1). Dans l'environnement du site de Springfield par exemple, le rapport d'activité est très proche de celui qui est observé dans l'environnement de Malvési (supérieur à 100), pour une activité en uranium du même ordre de grandeur. Ainsi, la synthèse des données de la littérature confirme le marquage de l'environnement terrestre des installations par l'uranium provenant des rejets, comme en témoigne l'excès de $1{ }^{\text {'238 }} \mathrm{U}$ par rapport au ${ }^{232} \mathrm{Th}$ (représentant le fond radiologique) enregistré par les plantes.

\section{Conclusion}

Les données acquises concernant l'activité des isotopes de l'uranium dans les plantes concourent à l'amélioration de la connaissance des sources de l'uranium dans l'environnement des sites du cycle du combustible : le fond radiologique et les rejets chroniques ou incidentels issus de ces sites. De plus, la normalisation de l'activité en ${ }^{238} \mathrm{U}$ par rapport à l'activité en ${ }^{232} \mathrm{Th}$, se révélant être une méthode pertinente pour identifier des rejets d'uranium naturel, est potentiellement utilisable dans l'environnement des sites miniers.

\section{Références}

AREVA (2010) Rapport annuel environnement année 2009-2010, Comurhex Malvési SECQ - Environnement, 33 p.

AREVA (2013a) Rapport annuel environnement année 2013, Comurhex Malvési SECQ - Environnement, 27 p.

AREVA (2013b) Données chiffrées et informations sur la sûreté nucléaire et la radioprotection du site AREVA Tricastin - Édition 2013, 63 p.

Bellis D., Ma R., McLeod C.W. (2001) Characterisation of airborne uranium and thorium contamination in Northern England through measurement of $\mathrm{U}$, Th and ${ }^{235} \mathrm{U} /{ }^{238} \mathrm{U}$ in tree bark, J. Environ. Monit. 3, 198-201.

Buchmann J.H., De Souza Sarkis J.E., Rodrigues C. (2000) Determination of metals in plant samples by using a sector field inductively coupled plasma mass spectrometer, Sci. Total Environ. 263, 221-229.

Howe S.E., Davidson C.M., McCartney M. (2002) Determination of uranium concentration and isotopic composition by means of ICP-MS in sequential extracts of sediment from the vicinity of a uranium enrichment plant, J. Anal. At. Spectrom. 17, 497-501. 
Jeambrun M., Pourcelot L., Mercat C., Boulet B., Pelt E., Chabaux F., Cagnat X., Gauthier-Lafaye F. (2012) Contribution of uranium, thorium and decay products in soil, water and atmospheric particles, to the activity concentration in lettuce and wheat samples, J. Environ. Monit. 14, 2902-2912.

Ketterer M.E., Hafer K.M., Link C.L., Royden C.S., Hartsock W.J. (2003) Anthropogenic ${ }^{236}$ U at Rocky Flats, Ashtabula river harbor, and Mersey estuary: Three case studies by sector inductively coupled plasma mass spectrometry, J. Environ. Rad. 67, 191-206.

Le Roux, G. (2007) Radionucléides naturels en France, approche géochimique de la variabilité des radionucléides naturels d'origine tellurique $\left({ }^{40} \mathrm{~K}\right.$ et chaînes U-Th) dans les sols et les sédiments, rapport IRSN/DEI/SESURE 2007-21, 41 p.

Lloyd N.S., Chenery S.R.N., Parrish R.R. (2009) The distribution of depleted uranium contamination in Colonie, NY, USA, Sci. Total Environ. 408, 397-407.

Pettersson H.B.L., Holm E. (1992) Investigation of aerial dispersion of uranium isotopes from a nuclear fuel fabrication facility, Waste Manag. 12, 85-97.

Pourcelot L., Boulet B., Cossonnet C. (2011a) Contribution des isotopes de l'uranium à l'expertise des sources de ce radioélément dans l'environnement, Radioprotection 46, 345-358.

Pourcelot L., Boulet B., Le Corre C., de Vismes Ott A., Cagnat X., Cossonnet C., Thomas S., Loyen J., Fayolle C., Van Hecke W., Martinez B., Petit J., Kaltenmeier R., Gieré R. (2011b) Actinides and decay products in some foodstuffs and bioindicators in the surrounding an uranium plant, J. Environ. Monit. 13, 1327-1336.

Pourcelot L., Boulet B., Le Corre C., Loyen J., Fayolle C., Tournieux D., Van Hecke W., Martinez B., Petit J. (2011c) Isotopic evidence of natural uranium and spent fuel uranium releases into the environment, J. Environ. Monit. 13, 355-361.
Pourcelot L., Masson O., Renaud P., Cagnat X., Boulet B., Cariou N., De Vismes-Ott A. (2015) Environmental consequences of uranium atmospheric releases from fuel cycle facility II. The atmospheric deposition of uranium and thorium on plants, J. Environ. Rad. 141, 1-7.

Rihs S., Prunier J., Thien B., Lemarchand D., Pierret M.-C., Chabaux F. (2011) Using short-lived nuclides of the U- and Th-series to probe the kinetics of colloid migration in forested soils, Geochim. Cosmochim. Acta 75, 7707-7724.

Taylor S.R. (1964) Abundance of chemical elements in the continental crust : a new table, Geochim. Cosmochim. Acta 28, 1273-1285.

Tortorello R., Widom E., Renwick W.H. (2014) Use of uranium isotopes as a temporal and spatial tracer of nuclear contamination in the environment, J. Environ. Rad. 124, 287-300.

United Nations Scientific Committee on Effects of Atomic Radiation UNSCEAR (2000) Report to the General Assembly. In: Sources and Effects of Ionizing Radiation, Vol. I. New York.

Wedepohl K.H. (1995) The composition of the continental crust, Geochim. Cosmochim. Acta 59, 1217-1232.

Yoshida S., Muramatsu Y., Tagami K., Uchida S. (1998) Concentrations of lanthanide elements, Th and $\mathrm{U}$ in 77 Japanese surface soils, Environ. Intern. 24, 275-286.

Yoshida S., Muramatsu Y., Tagami K. (2001) Determination of uranium isotopes in soil core samples collected on the JCO grounds after the criticality accident, Environ. Sci. Tech. 35, 4174-4179.

Zoriy P., Ostapczuk P., Dederichs H., Hobig J., Lennartz R., Zoriy M. (2010) Biomonitoring of environmental pollution by thorium and uranium in selected regions of the Republic of Kazakhstan, J. Environ. Rad. 101, 414-420.

Cite this article as: L. Pourcelot, B. Boulet, N. Cariou. Utilisation des isotopes de l'uranium et du rapport U/Th pour évaluer le marquage des végétaux consécutif aux rejets d'uranium des installations du cycle du combustible. Radioprotection 50(3), 209-214 (2015). 